Research Article

\title{
The Ensemble Machine Learning-Based Classification of Motor Imagery Tasks in Brain-Computer Interface
}

\author{
Abdulhamit Subasi $\mathbb{i D}^{1,2}$ and Saeed Mian Qaisar $\mathbb{D}^{2,3}$ \\ ${ }^{1}$ Institute of Biomedicine, Faculty of Medicine, University of Turku, Kiinanmyllynkatu 10, Turku 20520, Finland \\ ${ }^{2}$ College of Engineering, Effat University, Jeddah 22332, Saudi Arabia \\ ${ }^{3}$ Communication and Signal Processing Lab, Energy and Technology Research Center, Effat University, \\ Jeddah 22332, Saudi Arabia \\ Correspondence should be addressed to Saeed Mian Qaisar; sqaisar@effatuniversity.edu.sa
}

Received 20 August 2021; Revised 30 September 2021; Accepted 25 October 2021; Published 9 November 2021

Academic Editor: G R Sinha

Copyright (c) 2021 Abdulhamit Subasi and Saeed Mian Qaisar. This is an open access article distributed under the Creative Commons Attribution License, which permits unrestricted use, distribution, and reproduction in any medium, provided the original work is properly cited.

\begin{abstract}
The Brain-Computer Interface (BCI) permits persons with impairments to interact with the real world without using the neuromuscular pathways. BCIs are based on artificial intelligence piloted systems. They collect brain activity patterns linked to the mental process and transform them into commands for actuators. The potential application of BCI systems is in the rehabilitation centres. In this context, a novel method is devised for automated identification of the Motor Imagery (MI) tasks. The contribution is an effective hybridization of the Multiscale Principal Component Analysis (MSPCA), Wavelet Packet Decomposition (WPD), statistical features extraction from subbands, and ensemble learning-based classifiers for categorization of the MI tasks. The intended electroencephalogram (EEG) signals are segmented and denoised. The denoising is achieved with a Daubechies algorithm-based wavelet transform (WT) incorporated in the MSPCA. The WT with the 5th level of decomposition is used. Onward, the Wavelet Packet Decomposition (WPD), with the 4th level of decomposition, is used for subbands formation. The statistical features are selected from each subband, namely, mean absolute value, average power, standard deviation, skewness, and kurtosis. Also, ratios of absolute mean values of adjacent subbands are computed and concatenated with other extracted features. Finally, the ensemble machine learning approach is used for the classification of MI tasks. The usefulness is evaluated by using the BCI competition III, MI dataset IVa. Results revealed that the suggested ensemble learning approach yields the highest classification accuracies of $98.69 \%$ and $94.83 \%$, respectively, for the cases of subject-dependent and subject-independent problems.
\end{abstract}

\section{Introduction}

A Brain-Computer Interface (BCI) allows individuals to use electroencephalogram (EEG) signals to operate external equipment such as virtual worlds, robots, or spelling machines. The fundamental objective of the $\mathrm{BCI}$ is to use brain signals to create the required commands to control peripherals. The most important application is to bypass injured areas of the body or stimulate partly paralyzed organs. BCI devices are viewed as the best solution to mitigate problems for persons with various neuromuscular impairments such as spinal cord damage, amyotrophic lateral sclerosis, cerebral palsy, and stroke [1].
BCI systems may be divided into two categories based on the EEG signals collection methods: noninvasive and invasive. Because of the ease of usage, much current research has focused on noninvasive BCIs. Event-related potentials, steady-state visual-evoked potentials, and slow cortical potentials are the three main noninvasive BCI approaches [2]. In noninvasive approach, different EEG signals can be utilized in BCI. Within the EEG alpha and beta frequency regions of the brain signals, BCI systems typically employ Motor Imagery approaches to produce event-related actions. This form of BCI is mostly utilized for cursor control on computer screens and wheelchair navigation or in virtual environments. Several Motor Imagery (MI) techniques are commonly used, including 
tongue movement, left/right hand movement, foot movement, and mental counting [3]. The goal of BCI technology is to assist people with brain diseases including cerebral palsy, amyotrophic lateral sclerosis, and motor neuron disease. EEG is commonly used as a tool for the BCI system $[4,5]$. Based on phenomena of event-related synchronization (ERS) and event-related desynchronization (ERD), scientists can interpret and identify MI-related brain signals. The translation of imagination to action involves ERS and ERD. Both ERD and ERS are presented by variations in the EEG signal's oscillatory behaviour and can be investigated by the time-frequency analysis to identify the MI tasks [6]. MI is characterized as a human brain's ability to resynthesize motor experiences with no obvious movement. Such mental images may both appear consciously and be created and controlled deliberately by a subject making MI, which is a flexible and usable method for examining processes of human cognition and motor activity. As various studies have shown, MI uses almost the same neural framework as motor execution, which enables motor activity to be altered by MI training. The MI-based BCI uses variations in the cortical sensorimotor rhythms (SMR), generally ERD related to the different sensorimotor events, including MI [7]. In addition, BCI may serve as a technical bridge for the management of Active and Assisted Living (AAL) systems in the sense of intelligent environments and smart homes. As with any other traditional AAL device interface, the consumer needs to view BCI-enabled control as simple and normal as possible in order to encourage BCI acceptance and effectiveness [8].

Computer-based automated MI signal detection is essential for providing continuous assistance to the intended patients. The preprocessing, feature extraction, dimension reduction, and classification are all parts of the EEG-based automated MI signal detection approaches $[9,10]$.

Feature extraction and dimension reduction are the most critical aspects of the classification system for EEG-based MI signals since they greatly affect classifier efficiency and computational complexity. If the features retrieved from EEG signals include irrelevant characteristics, the classifier's performance will suffer. The amount of features determines the classifier's processing cost. As a result, extracting the appropriate amount of relevant features from EEG-based MI signals is critical for achieving high classification performance and computational effectiveness for a classifier [9]. In this study, the dataset IVa from the BCI competition is utilized in the experiments [11]. AA, AL, AV, AW, and AY are codes of five healthy participants that contributed to this dataset. Two classes of MI activities, right hand and right foot movement, referred to as class 1 and class 2, respectively, are involved.

1.1. Contribution. The main objective of this work is to extract relevant features from the EEG signals and to design a classifier that can effectively recognize the intended MI tasks.

The major contributions are to propose a novel hybridization of the Multiscale Principal Component Analysis (MSPCA), Wavelet Packet Decomposition (WPD), subbands statistical features selection, and ensemble learning technique for automated classification of the MI tasks. The functional steps are as follows:

(i) The Multiscale Principal Component Analysis (MSPCA) is used for denoising.

(ii) The Wavelet Packet Decomposition is used for producing the subbands.

(iii) The six different statistical features are extracted from each subband. These are mean absolute value, power, standard deviation, skewness, kurtosis, and ratio of absolute mean values of adjacent subbands.

(iv) The extracted features are passed to the proposed ensemble learning-based classifiers for automated identification of the MI tasks.

1.2. Organization. The remainder of the paper is organized as follows. Section 2 presents a literature review. In Section 3, materials and methods are introduced, Section 4 discusses the results, and the conclusion is presented in Section 5.

\section{Literature Review}

The loss of motor function is one of the most concerning effects of injury or disease to the nervous system. The BCI assistive technologies have allowed artificial prostheses, wheelchairs, and computers to be controlled by the electrical activity of the brain in this decade. The major challenges in the BCI systems are precision and processing effectiveness. The current systems have high computational complexity and need advanced and resourceful processing systems to attain a real-time response. Additionally, their classification performance and robustness need to be improved. In this context, several studies have been presented [12, 13].

Zarei et al. [9] used a combination of the Principal Component Analysis (PCA) and the cross-covariance (CCOV) method for features extraction from the EEG signals for the BCI application. The multilayer perceptron neural networks (MLP) and Least Square Support Vector Machine (LS-SVM) are used for classification. The performance of the system is tested by using the BCI competitions dataset IVa. Kayikcioglu and Aydemir [10] extracted features from the EEG signals by using two-dimensional features mining from the 2nd order polynomial coefficients. Then, the functions are categorized using the algorithm $\mathrm{k}$-nearest neighbor (k-NN). They achieved considerable enhancement in speed and accuracy while evolving the performance for the dataset Ia from the 2003 BCI competition. Leamy et al. [12] conducted a comparative experimental research, from a machine learning perspective, for MI-related EEG features in stroke subjects. They try to explore if such features are generalizable to use trained machine learning parameters employing healthy subjects and stroke-affected patients. If BCI is trained with appropriate data, it gives relatively good results to stroke patients; then such a deployment model will make BCI far more realistic in a clinical setting for stroke recovery. On the other hand, if the stroke-affected EEG is significantly different 
from healthy EEG or changes over time, it may need more sophisticated architecture from a machine learning perspective for the realistic implementation of BCI in such a setting.

Li et al. [13] proposed a new approach for MI pattern identification. It combines a common spatial pattern algorithm for frequency band selection and features selection, and the classification is carried out with the particle swarm optimized twin Support Vector Machine. They used datasets IIb of BCI competition IV to test the proposed system. For a classification task, Kevric and Subasi [14] employed MSPCA-based denoising of the EEG signals. Comparison among three features extraction techniques, namely, the Empirical Mode Decomposition (EMD), Discrete Wavelet Transform (DWT), and WPD, is conducted. The extracted features sets are classified by using the k-Nearest Neighbor (k-NN) algorithm. The system performance is tested by using the publicly available BCI competition III dataset IVa. Miao et al. [15] have suggested an EEG signals channel selection method. It uses the linear discriminant criteria for automated selection of channels with strong discriminative capabilities. Furthermore, the artificial bee colony algorithm is used for dimension reduction. The performance is tested by using the dataset IVa from the BCI competition III. In [16], Baali et al. have used a signal-dependent orthogonal transformation for features extraction. The classification is carried out by using a tree-based logistic model classifier. In [17], Chaudhary et al. used the flexible analytic wavelet transform (FAWT) for features extraction. The classification is carried out with ensemble learning-based subspace k-Nearest Neighbor (k-NN) classifier. In [18], Rahman et al. have used the Rényi min-entropy-based features extraction approach. The extracted features are used for classifying 4 different BCI categories by using the Random Forest (RF) algorithm. The performance of the proposed method is evaluated by using the BCI competition IV dataset.

Khare and Bajaj [19] employed the extreme learning machine-based classification of the MI tasks. The channels selection is realized by using the multicluster unsupervised learning approach. The signal decomposition is performed by using a flexible variational mode decomposition (FVMD). Pertinent features from different modes are explored, namely, hjorth, entropy, and quartiles. In [20], the authors have used the flexible analytic wavelet transform (FAWT) for signal decomposition. Time-frequency attributes are calculated from subbands. The PCA, kernel PCA (KPCA), locally linear embedding (LLE), and Laplacian Eigenmaps (LE) are used for feature selection. The Linear Discriminant Analysis (LDA) algorithm is used for the classification. The performance is tested by using the BCI competition III dataset IIIb.

Tiwari et al. [21] proposed a Deep Neural Network (DNN) model for automated identification of the MI tasks by utilizing the EEG signals. The Power Spectral Densities (PSDs) are extracted as features from subbands by applying a bank of Butterworth filters. The performance is tested for the BCI competition III and V dataset MI tasks. Musallam et al. [22] utilized a Convolutional Neural Network (CNN) model that incorporates a number of different methods, including temporal convolutional networks (TCNs), separable convolution, depthwise convolution, and layer fusion. The intended EEG signals are processed by two successive 1D convolution stages. The first in the time domain and subsequently channelwise and the second based on the imagelike representation are used as an input of the main TCN. The performance is tested by using the BCI competition IV, IIa dataset.

\section{Materials and Methods}

The proposed system's framework is shown in Figure 1. A description and parameterization of different system modules are given in the following section.

3.1. Dataset. The suggested system performance is evaluated by using the well-known BCI competition III, dataset IVa ${ }^{1}$ [11]. AA, AL, AV, AW, and AY are codes of five healthy participants that contributed to this dataset. They completed two classes of MI activities involving right hand and right foot movement, referred to as class 1 and class 2, respectively. Subjects are seated in comfortable chairs with armrests. The EEG signals are acquired from 118 electrodes, mounted by following the 10/20 globally accepted standard. Each considered subject performed 140 trials of each category. Being two considered classes of tasks, it resulted in a total of 280 trials per subject. Each trial is carried out for a duration of $3.5 \mathrm{sec}$. For each category, the data is made up of different-sized training and testing sets.

The training set for subjects $\mathbf{A A}, \mathbf{A L}, \mathbf{A V}, \mathbf{A W}$, and $\mathbf{A Y}$ has $168,224,84,56$, and 28 trials, respectively. The testing set consists of 112, 56, 196, 224, and 252 trials for participants AA, AL, AV, AW, and AY, respectively.

The EEG signals are originally recorded at a rate of $1 \mathrm{kHz}$. These EEG signals are bandlimited to $50 \mathrm{~Hz}$ by using digital filtering and are onward downsampled to the rate of $100 \mathrm{~Hz}$ [11]. These downsampled versions of signals are used in this study. The EEG signals from only three channels $(\mathrm{C} 3, \mathrm{Cz}$, and C4) are selected from a total of 118 available channels. This is because these channels contain the most discriminatory features on Motor Imagery activities involving the hands and feet. For each patient, 280 EEG segments of 3.5 seconds, with 3 selected channels, are prepared [11]. These are from two categories: right hand and foot. In total, 1400 EEG instances were used for the five mentioned subjects. They belong to the two considered classes of the MI tasks.

3.2. Denoising with Multiscale Principal Component Analysis (MSPCA). In multivariate statistical analysis, the PCA is one of the most important models. Let a measurement dataset with $m$ sensors exist, such as $x \in R^{m}$. Each sensor in the measurement sample contains $n$ separate sampling data, which are combined into a data matrix of size mxn. The process is given by

$$
X=\left[x_{1}, x_{2}, x_{3}, \ldots, x_{n}\right] .
$$

Each row of $X$ represents a sample, and each column represents a measurement variable. The PCA model begins 


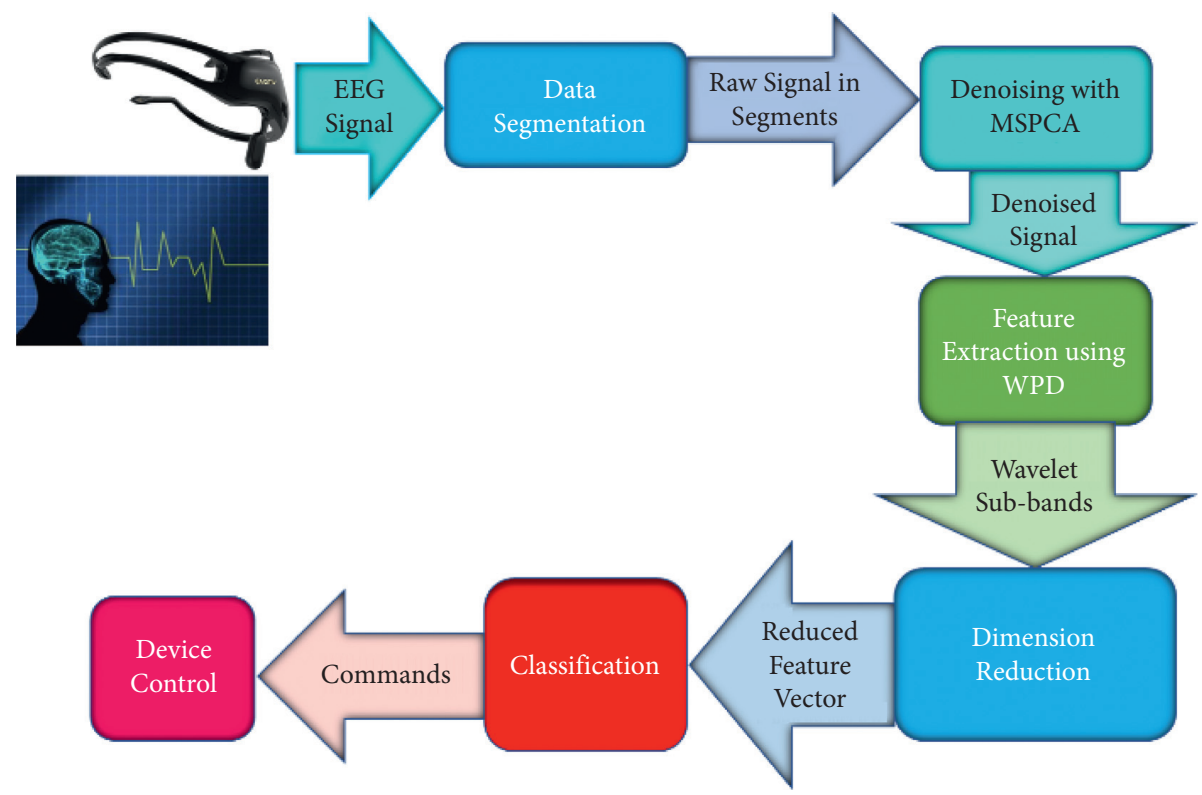

Figure 1: The system block diagram.

by standardizing each sample of $X$ by computing the covariance matrix of $X$. The process is given by

$$
\operatorname{cov}(x) \approx \frac{X^{T} \cdot X}{n-1} .
$$

The size of the feature values is ordered from large to small when the feature decomposition of $X$ is done. The process of decomposing $X$ in its principal components is given by equation (3), where $P \epsilon R^{m \times A}$ contains first $A$ feature vectors of $\operatorname{cov}(x) . T \epsilon R^{n \times A}$ is a matrix, where each column is known as the principal element variable. $A$ is the count of principal components, and it is equal to the number of columns in $T$.

$$
\left\{\begin{array}{l}
X=\widehat{X}+E r=T . P^{T}+E r, \\
T=X . P .
\end{array}\right.
$$

Equation (4) can be used to determine the principal component's covariance, where $\lambda_{1}, \lambda_{2}, \ldots, \lambda_{n}$ are the first $A$ large eigenvalues of the covariance matrix of $X$.

$$
\Lambda=\frac{X^{T} \cdot X}{n-1}=\left[\begin{array}{c}
\lambda_{1} \ldots \ldots \cdot \\
\ldots \cdot \lambda_{2} \ldots \cdot \\
\cdot \\
\ldots \\
\ldots \ldots \cdot \lambda_{n}
\end{array}\right] .
$$

In this paper, the wavelet transform is combined with the Principal Component Analysis (PCA) to create MSPCA for the incoming signal denoising purpose. The principle of wavelet transform is described in Section 3.3. In this study, the 5 th level of decomposition is realized by using the
Daubechies wavelet analysis algorithm [23]. MATLAB is used for implementing the wavelet transform [24].

The ability of standard PCA is enhanced by incorporating the multiscale analysis. Collectively, it results in the multiscale PCA (MSPCA) [25]. In MSPCA, the PCA's capacity to extract covariance between variables is combined with orthonormal wavelets' ability to distinguish deterministic features from stochastic processes and approximately decorrelate the autocorrelation across observations. It identifies linearly related wavelet coefficients at multiple level subbands, obtained with wavelet transform. It allows representing each considered subband with fewer features while removing the autocorrelated coefficients. It results in a simplified representation of the considered subbands at each level of decomposition. The EEG waveforms are decomposed by using the Daubechies wavelet analysis algorithm with the 5th level of decomposition. In the next step, the PCA of detailed coefficients, obtained at each level, is utilized to select the principal components at each scale. Onward, the signal is reconstructed by using the wavelet synthesis. It diminishes the unwanted noise from the incoming signal and generates a simple and noise-free signal version $[25,26]$. MATLAB is used for implementing the MSPCA [24].

\subsection{Features Extraction with Wavelet Packet Decomposition} (WPD). Wavelets are well-known functions and widely used for multiresolution time-frequency analysis. Wavelets can be mathematically described by equation (5) [23], where the dilation parameter is represented by $s$ and the translation parameter is represented by $u$. The parameters can be generated at the same time with different frequencies.

$$
\psi(t)=\frac{1}{\sqrt{S}} \psi\left(\frac{(t-u)}{s}\right) .
$$


The process of decomposing a signal $x(t)$, by using wavelet transform, can be given by

$$
W_{X}(u, s)=\frac{1}{\sqrt{S}} \int_{-\infty}^{+\infty} x(t) \psi^{*}\left(\frac{(t-u)}{s}\right) \mathrm{d} t .
$$

A discrete version of the wavelet transform (DWT) is used in this study. The selection of the right number of wavelet decomposition levels, $m$, is the first key step in the DWT decomposition. The incoming signal $x[n]$ passes concurrently through both the high-pass and low-pass filters, $h[k]$ and $l[k]$. For the $m^{\text {th }}$ scale level, the output is represented by two subbands, namely, Detail $\left(D_{m}\right)$ and Approximation $\left(A_{m}\right)$. The process is clear from equations (7) and (8), where $H$ is the order of filters used at different decomposition stages:

$$
\begin{gathered}
D_{m}[k] \sum_{k=1}^{H} x[n] \cdot h[2 \cdot k-n], \\
A_{m}[k] \sum_{k=1}^{H} x[n] . l[2 \cdot k-n] .
\end{gathered}
$$

The Wavelet Packet Decomposition is known as the extension of Discrete Wavelet Transform (DWT). The DWT mainly focuses on the low-frequency components, known as approximate coefficients. However, WPD utilizes both approximate and detailed coefficients, high-frequency components [27]. Consequently, when tactfully used, the WPD can result in signal decomposition with superior frequency resolution compared to the DWT [26]. In the studied case, the denoised signal is further analysed by using four levels of WPD. Pertinent statistical features are extracted from multiresolution subbands, obtained with the 4 th level of WPD. MATLAB is used for implementing the WPD [24]. The principle of employed WPD with the 4 th level of decomposition is shown in Figure 2, where $D_{m}$ and $A_{m}$ are, respectively, detailed and approximation coefficients at different decomposition stages and $m \in\{1,2,3,4\}$.

3.4. Dimension Reduction. Since the dimension of the extracted features with WPD is high, the dimension should be reduced. Therefore, in this study, the dimension of extracted features is reduced by using statistical values of the WPD subbands. Using the statistical values of the subbands, the pertinent classifiable features are created from 16 subbands, shown in Figure 2. Five features are extracted from each subband, namely, mean absolute value, average power, standard deviation, skewness, and kurtosis. It results in $16 \times$ $5=80$ features. Additionally, the ratios of absolute mean values of the adjacent subbands are computed, resulting in 15 more features. In this way, in total, 95 features are extracted for each EEG instance, resulting in feature set dimension of $1400 \times 95$ for all considered instances.

3.5. Classification Methods. The prepared features set is categorized by using k-Nearest Neighbor (k-NN), C4.5 Decision Tree, REP Tree, Support Vector Machine (SVM),
Random Tree (RT), and RF, which are all well-known robust classification algorithms. Weka is used for evaluating the considered classifiers $[28,29]$. To avoid any bias in findings due to the limited volume of the dataset, the 10 -fold crossvalidation $(10-\mathrm{CV})$ approach is used along with multiple evaluation measures, namely, accuracy, F-measure, and the area under the ROC curve (AUC). Here, ROC stands for receiver operating characteristic [29].

\subsubsection{Support Vector Machine (SVM). The SVM searches for} hyperplane in an $\mathrm{N}$-dimensional space in the classification of the data points. The SVM can be used for both classification and regression. The system functions by focusing on the decision line. It is a theoretically mature algorithm, only takes tens of instances for training, and is unaffected by the number of dimensions. Furthermore, effective approaches are developed to rapidly train this classifier [30]. In this study, the SVM is used with the cubic polynomial kernel and with a regularization parameter of 100 .

3.5.2. K-Nearest Neighbor $(k-N N)$. The k-NN refers to a supervised learning algorithm used in regression and classification problems. The algorithm functions by assuming that every data falling near each other belongs to the same class. It means that the algorithm considers that the classification of information is based on similarities. The technique is highly preferred because of its simplicity [30]. In this study, the $\mathrm{k}-\mathrm{NN}$ with $k=1$ is used. Here, $k$ is the number of neighbors, used in the decision.

3.5.3. REP Tree. REP Tree creates a decision or regression tree using information variance reduction and then prunes it using reduced-error pruning. It optimizes speed by only sorting values for numeric attributes once. The minimum number of instances per leaf, maximum tree depth, minimum fraction of training set variance for a split, and the number of folds for pruning are adjustable parameters [31]. In this study, the REP Tree is used with its default configurations, available in Weka $[28,29]$.

3.5.4. C4.5 Decision Tree. The C4.5 can create classifiers that are redescribed as rulesets. C4.5 starts by growing an initial tree using the divide-and-conquer method. It labels the potential test instances by using two heuristic criteria. The first is the information gain, which tries to minimize the total entropy of subsets. The second is the default gain ratio, which tries to divide the information gain by the information supplied via the test outcomes [30]. In this study, the C4.5 is used with its default configurations, available in Weka $[28,29]$.

3.5.5. Random Tree (RT) Classifiers. The RT is a supervised learning algorithm that is easy to use and flexible. The algorithm produces excellent results despite lacking hyperparameter tuning. A combination of decision subtrees is trained based on the bagging method. The primary concept 


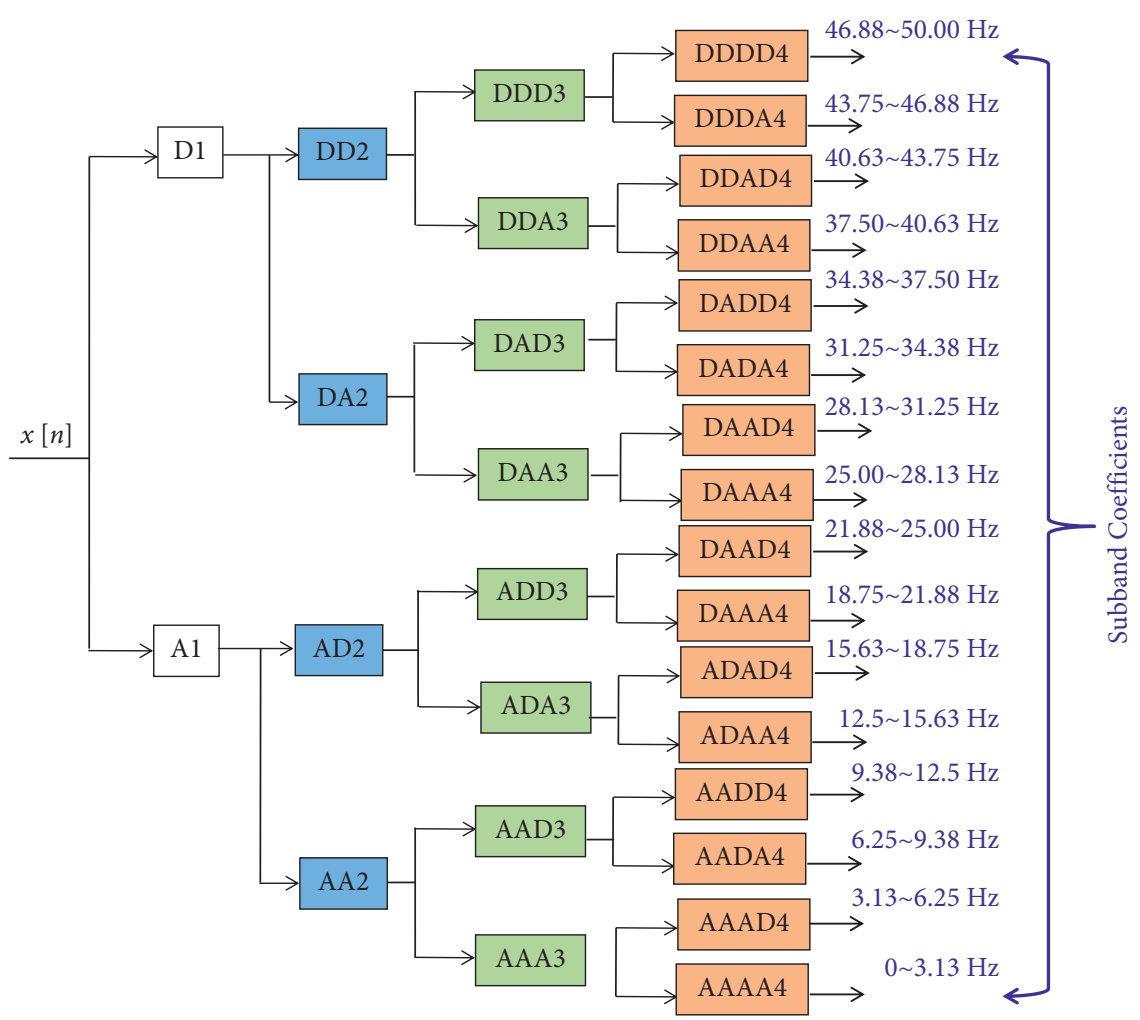

Figure 2: The employed WPD scheme.

of the functioning of the Random Tree is that combined learning models will increase the quality of results gained [31]. In this study, the RT is used with its default configurations, available in Weka $[28,29]$.

3.5.6. Random Forests (RF). The RF refers to a robust machine learning algorithm for various tasks such as classification and regression. The algorithm works by using bagging and randomness when creating each of the trees. It makes an uncorrelated forest of trees where their prediction is more accurate than a single tree [32]. In this study, the RF is used with 100 trees.

3.5.7. Rotation Forest (RoF). The RoF is a feature extractionbased classifier ensemble. We make the training data for a basic classifier by randomly partitioning the feature set into $Q$ subgroups. PCA is applied to each subgroup, and $Q$ is a parameter of the method. To retain the data's variability information, all basic components are kept. As a result, rotating the Q-axis produces additional attributes for a base classifier [33]. All primary components are kept in order to preserve the data's variability information. As a result, new features for a base classifier are formed by rotating the Q-axis [33]. The purpose of the rotation approach is to enhance individual accuracy while also providing variation within the group. Each base classifier's feature extraction contributes to diversity.

3.5.8. The Random Subspace Method (RSM). A well-known ensemble technique is the RSM [34]. The training data is also modified in the RSM. This change, however, is done in the feature space. The $B$-dimensional random subspace of the original $B$-dimensional feature space is thus obtained. As a result, the updated training set has $B$-dimensional training objects in it. Then, in the final decision rule, classifiers can be built in random subspaces and combined using simple majority voting [35].

3.6. The Ensemble Learning Method. The ensemble learning methods can improve the performance of classification [26]. In this framework, the RoF and the RSM classifiers are employed with single classifiers. Multiple classifiers are used for the considered classification task. Findings of classifiers with various accuracies are combined via an ensemble-based approach [36]. The principle is depicted with the help of Figure 3.

For the case of RoF, by randomly splitting the features set into $Q$ subgroups, we generate training data for a base classifier. After that, the PCA is applied to each subgroup. To maintain the data's variability information, all principal components are taken into consideration. This is how $Q$-axis rotations are realized to prepare new features for a base classifier. The rotation technique is designed to enhance individual accuracy while simultaneously fostering variation within the ensemble. Each base classifier's diversity is created by feature extraction. In this scenario, accuracy is measured by training each base classifier with the entire dataset [33].

For the case of RSM, the $B$-dimensional random subspace of the original features set was produced. As a result, the training set comprises $B$-dimensional training objects. In this approach, we built classifiers in random subspaces and used simple majority voting to aggregate their results [35]. 


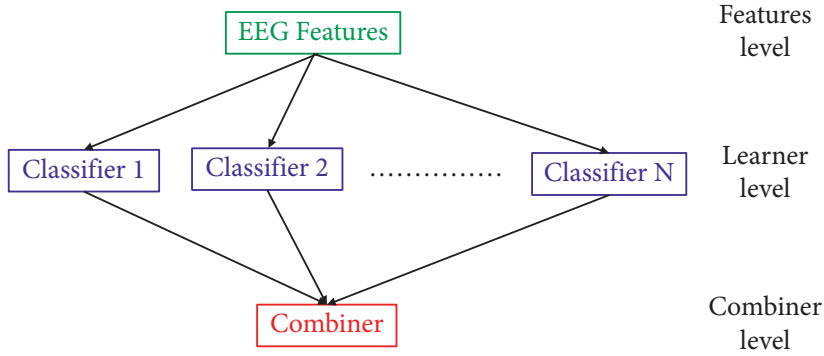

Figure 3: The general framework of ensemble classifiers.

3.7. Performance Evaluation Measures. In order to avoid any bias in the classification performance evaluation, multiple evaluation measures, namely, accuracy, F-measure, and AUC, are utilized [29]. The accuracy is defined by equation (9). True positives, true negatives, false positives, and false negatives are represented as $\mathrm{tp}, \mathrm{tn}, \mathrm{fp}$, and $\mathrm{fn}$, respectively. The F-measure is given by equation (10). The AUC presents the classification performance graphically. It is the area under the curve of the graph, obtained by tracing the True Positive Rate (TPR) with respect to the False Positive Rate (FPR). The TPR and FPR are, respectively, given by equations (11) and (12).

$$
\begin{aligned}
\operatorname{accuracy}(\mathrm{ACC}) & =\frac{t p+t n}{t p+t n+f p+f n} \times 100, \\
F-\text { measure } & =\frac{t p}{t p+1 / 2(f p+f n)}, \\
\mathrm{TPR} & =\frac{t p}{(t p+f n)}, \\
\mathrm{FPR} & =\frac{f p}{(f p+t n)} .
\end{aligned}
$$

\section{Results}

The system performance is tested by using the BCI competition III, dataset IVa [11]. An example of the input EEG signal and its denoised version, obtained with the MSPCA, is shown in Figure 4.

The denoised signal is onward decomposed in 16 subbands by using the 4 th level of WPD. An example of obtained subbands is shown in Figure 5.

The overall system performance is studied in terms of classification precision. Findings are outlined in Table 1. These results are also presented graphically. In Figure 6, the accuracy scores, obtained with different classifiers, are shown. Figures 7 and 8, respectively, show the F-Measure and AUC values, obtained with different classifiers.

It is evident from Table 1 that the ensemble of $\mathrm{k}-\mathrm{NN}$ and RoF attains the superior classification performance in most of the cases, compared to the other studied classifiers. The obtained percentages accuracies obtained for subjects AA, AL, AV, AW, and AY are, respectively, 96.67\%, 94.05\%, $89.64 \%, 96.43 \%$, and $90.71 \%$. However, the results are different for the case of subject AY. For AY, the highest classification accuracy of $98.69 \%$ is attained RSM with RF and $98.45 \%$ is attained by the RSM with C4.5. The RoF with C4.5 is the third with an accuracy of $97.14 \%$. RSM with RT is the fourth one with an accuracy of $97.02 \%$. RSM with $\mathrm{k}-\mathrm{NN}$ is the eighth with an accuracy of $92.38 \%$.

While considering the case of each subject, the highest accuracy of $98.69 \%$ is achieved by the RSM with RF. However, for all five subjects, the highest classification accuracy of $94.83 \%$ is achieved by the RoF with k-NN. It shows that, in general, the used assembly of MSPCA, WPD, and statistical feature selection using RoF with $\mathrm{k}-\mathrm{NN}$ results in the best classification performance for the studied dataset.

\section{Discussion}

The results, outlined in the above section, show that, for most of the cases, the proposed framework of MSPCA, WPD, statistical features selection, and RoF with k-NN leads towards a high classification accuracy. However, the best results obtained for the subject $\mathbf{A Y}$ are obtained for a combination of MSPCA, WPD, and statistical feature selection using RSM with RF. It happens due to the variation in EEG signals magnitudes and response time of subjects while executing an MI task. It has an impact on the shape of EEG signals as well as the performance of the postsegmentation, denoising, feature extraction, and classification algorithms. Therefore, various subjects have varying classification accuracy as a result of this.

The BCI is a well-explored domain, and making a performance comparison with state of the art is a tedious task. It is mainly because of the variety of datasets, preprocessing, features extraction, dimension reduction, and classification techniques used in the previous studies. However, a performance comparison of the suggested framework is made with state-of-the-art solutions using similar datasets. Table 2 provides a review of those studies. It indicates that the suggested method ensures a comparable or superior performance as compared to the previously presented methods. It indicates that the devised denoising, dimension reduction, and ensemble classification approaches have a substantial influence on the overall precision and performance of the system. The self-configurability of ensemble classifiers, as a function of the utilized training dataset, is one of their main advantages. The use of eventdriven tools can help in enhancing the computational effectiveness of the suggested method [45-48]. In the future, 


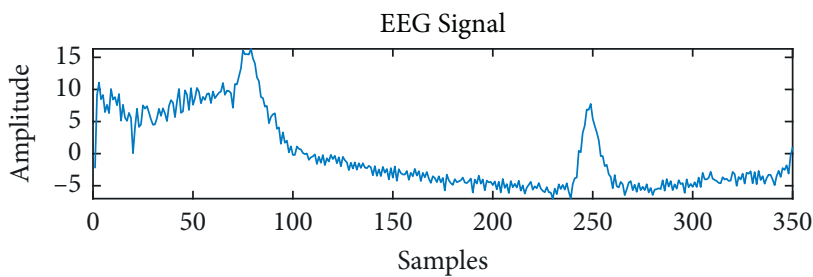

(a)

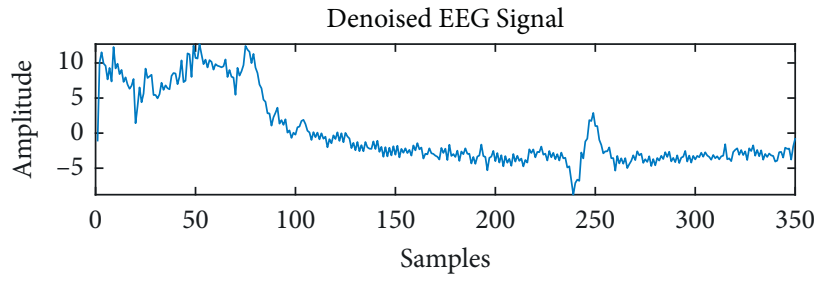

(b)

Figure 4: (a) The EEG signal and (b) denoised version of EEG signal.
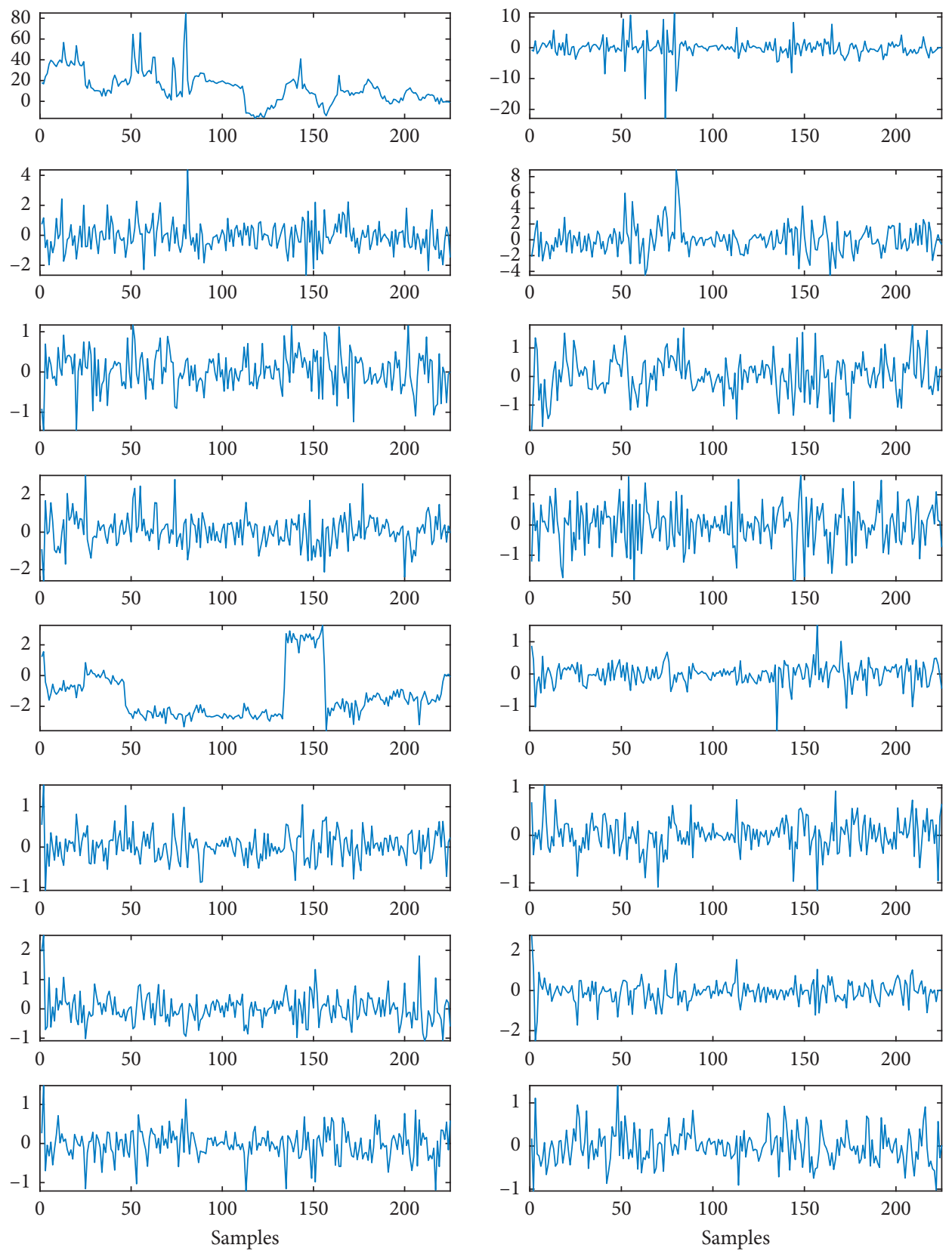

FIgURe 5: The 16 subbands, obtained with WPD. 
TABLE 1: Summary of the classification performance measures.

\begin{tabular}{|c|c|c|c|c|c|c|c|}
\hline \multirow[t]{2}{*}{ Subj. } & \multirow[t]{2}{*}{ Classifier } & \multicolumn{2}{|c|}{ Accuracy } & \multicolumn{2}{|c|}{ F-measure } & \multicolumn{2}{|c|}{ AUC } \\
\hline & & RSM & RoF & RSM & RoF & RSM & RoF \\
\hline \multirow{6}{*}{ AA } & SVM & 89.64 & 90.60 & 0.896 & 0.906 & 0.958 & 0.929 \\
\hline & $\mathrm{k}-\mathrm{NN}$ & 93.45 & 96.67 & 0.935 & 0.967 & 0.988 & 0.985 \\
\hline & $\mathrm{RF}$ & 82.02 & 84.76 & 0.820 & 0.848 & 0.900 & 0.928 \\
\hline & C4.5 & 67.50 & 74.76 & 0.675 & 0.748 & 0.764 & 0.834 \\
\hline & REP tree & 69.88 & 67.50 & 0.699 & 0.675 & 0.765 & 0.746 \\
\hline & RT & 70.83 & 74.29 & 0.706 & 0.742 & 0.799 & 0.827 \\
\hline \multirow{6}{*}{ AL } & SVM & 86.43 & 84.88 & 0.864 & 0.849 & 0.923 & 0.879 \\
\hline & $\mathrm{k}-\mathrm{NN}$ & 92.26 & 94.05 & 0.923 & 0.940 & 0.970 & 0.968 \\
\hline & RF & 75.00 & 77.26 & 0.750 & 0.773 & 0.830 & 0.852 \\
\hline & C4.5 & 71.07 & 73.69 & 0.711 & 0.737 & 0.786 & 0.812 \\
\hline & REP tree & 68.93 & 68.69 & 0.689 & 0.687 & 0.751 & 0.756 \\
\hline & $\mathrm{RT}$ & 70.95 & 70.60 & 0.708 & 0.703 & 0.778 & 0.786 \\
\hline \multirow{6}{*}{ AV } & SVM & 87.02 & 84.40 & 0.870 & 0.844 & 0.928 & 0.886 \\
\hline & $\mathrm{k}-\mathrm{NN}$ & 88.93 & 89.64 & 0.889 & 0.896 & 0.955 & 0.939 \\
\hline & $\mathrm{RF}$ & 73.69 & 76.90 & 0.737 & 0.769 & 0.818 & 0.857 \\
\hline & C4.5 & 61.90 & 71.31 & 0.619 & 0.713 & 0.677 & 0.767 \\
\hline & REP tree & 62.38 & 66.19 & 0.624 & 0.662 & 0.671 & 0.721 \\
\hline & RT & 65.00 & 65.95 & 0.644 & 0.654 & 0.691 & 0.738 \\
\hline \multirow{6}{*}{ AW } & SVM & 86.90 & 85.00 & 0.869 & 0.850 & 0.926 & 0.876 \\
\hline & $\mathrm{k}-\mathrm{NN}$ & 94.64 & 96.43 & 0.946 & 0.964 & 0.983 & 0.976 \\
\hline & $\mathrm{RF}$ & 77.02 & 78.81 & 0.770 & 0.788 & 0.844 & 0.867 \\
\hline & C4.5 & 67.62 & 71.19 & 0.676 & 0.712 & 0.727 & 0.788 \\
\hline & REP tree & 63.45 & 68.33 & 0.634 & 0.683 & 0.683 & 0.742 \\
\hline & $\mathrm{RT}$ & 68.69 & 70.24 & 0.685 & 0.700 & 0.735 & 0.780 \\
\hline \multirow{6}{*}{ AY } & SVM & 92.02 & 91.90 & 0.920 & 0.919 & 0.984 & 0.942 \\
\hline & $\mathrm{k}-\mathrm{NN}$ & 92.38 & 90.71 & 0.924 & 0.907 & 0.977 & 0.952 \\
\hline & $\mathrm{RF}$ & 98.69 & 94.29 & 0.987 & 0.943 & 0.999 & 0.991 \\
\hline & C4.5 & 98.45 & 97.14 & 0.985 & 0.971 & 0.999 & 0.995 \\
\hline & REP tree & 95.60 & 93.10 & 0.956 & 0.931 & 0.988 & 0.980 \\
\hline & $\mathrm{RT}$ & 97.02 & 93.10 & 0.970 & 0.931 & 0.996 & 0.985 \\
\hline \multirow{6}{*}{ ALL } & SVM & 89.98 & 90.12 & 0.900 & 0.901 & 0.962 & 0.932 \\
\hline & $\mathrm{k}-\mathrm{NN}$ & 93.55 & 94.83 & 0.935 & 0.948 & 0.984 & 0.974 \\
\hline & $\mathrm{RF}$ & 80.36 & 81.83 & 0.804 & 0.818 & 0.889 & 0.901 \\
\hline & C4.5 & 68.57 & 73.00 & 0.686 & 0.730 & 0.769 & 0.815 \\
\hline & REP tree & 68.60 & 69.02 & 0.686 & 0.690 & 0.765 & 0.760 \\
\hline & RT & 72.57 & 72.40 & 0.724 & 0.722 & 0.807 & 0.809 \\
\hline
\end{tabular}

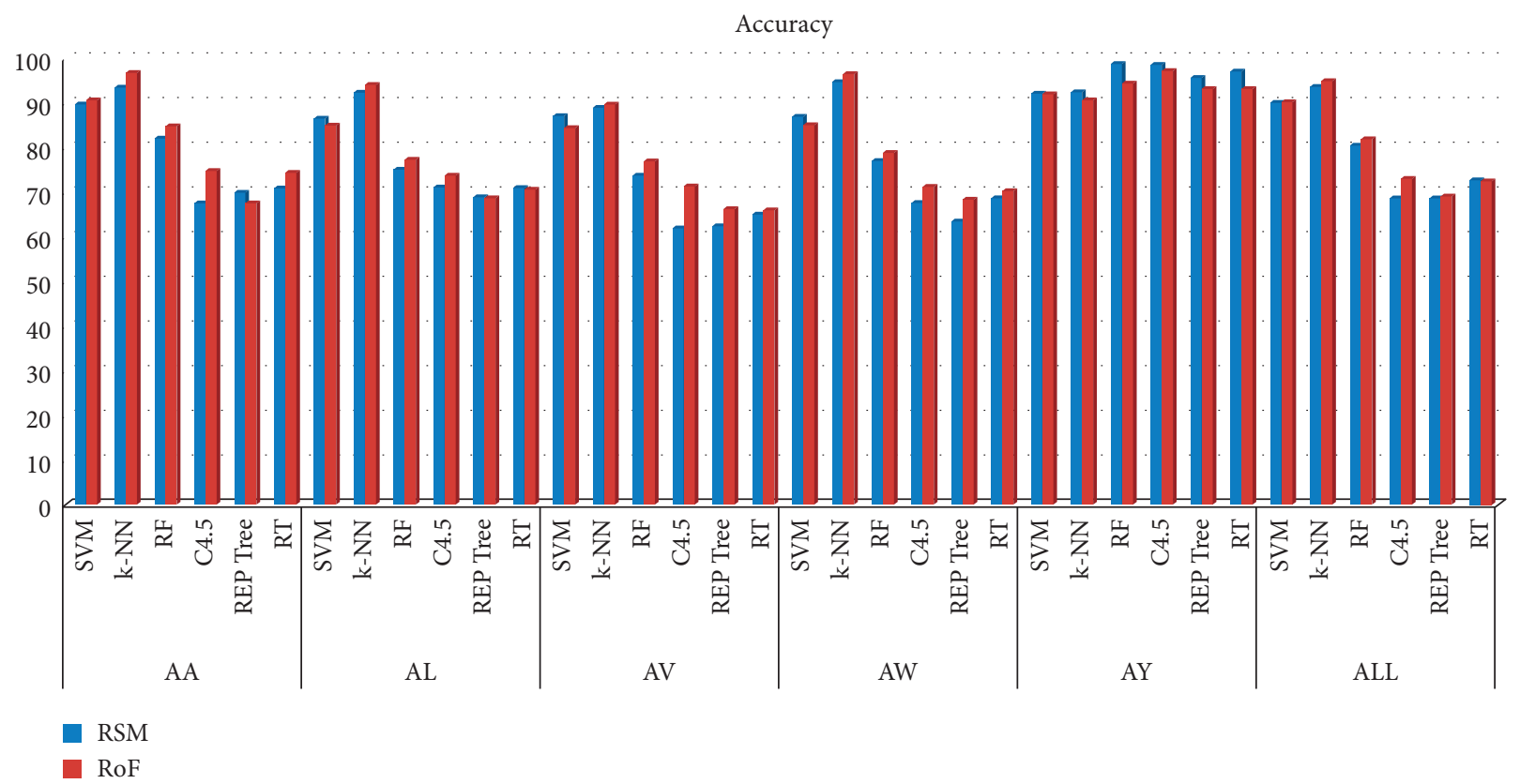

Figure 6: Accuracy of different classifiers. 


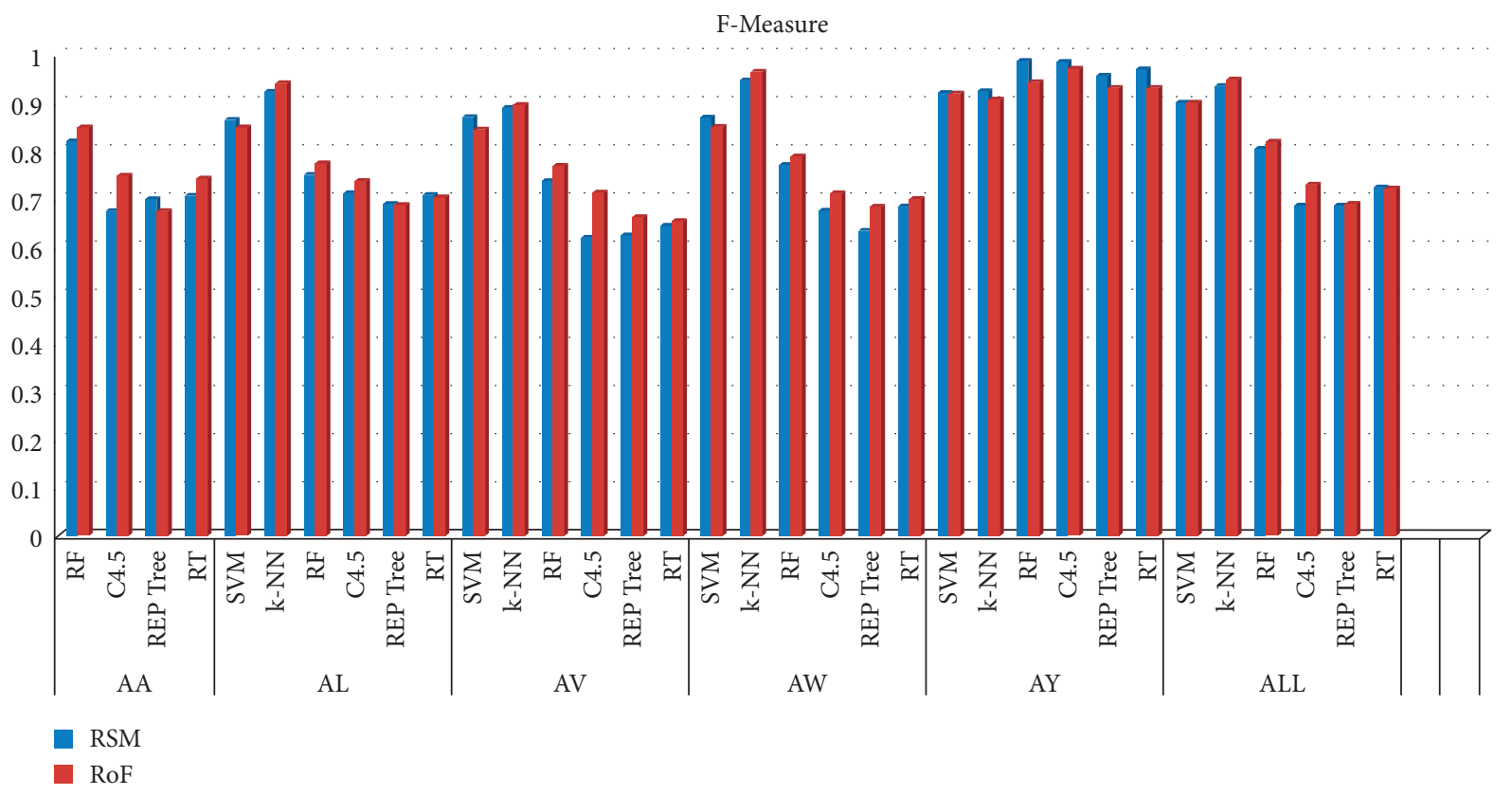

Figure 7: F-measure of different classifiers.

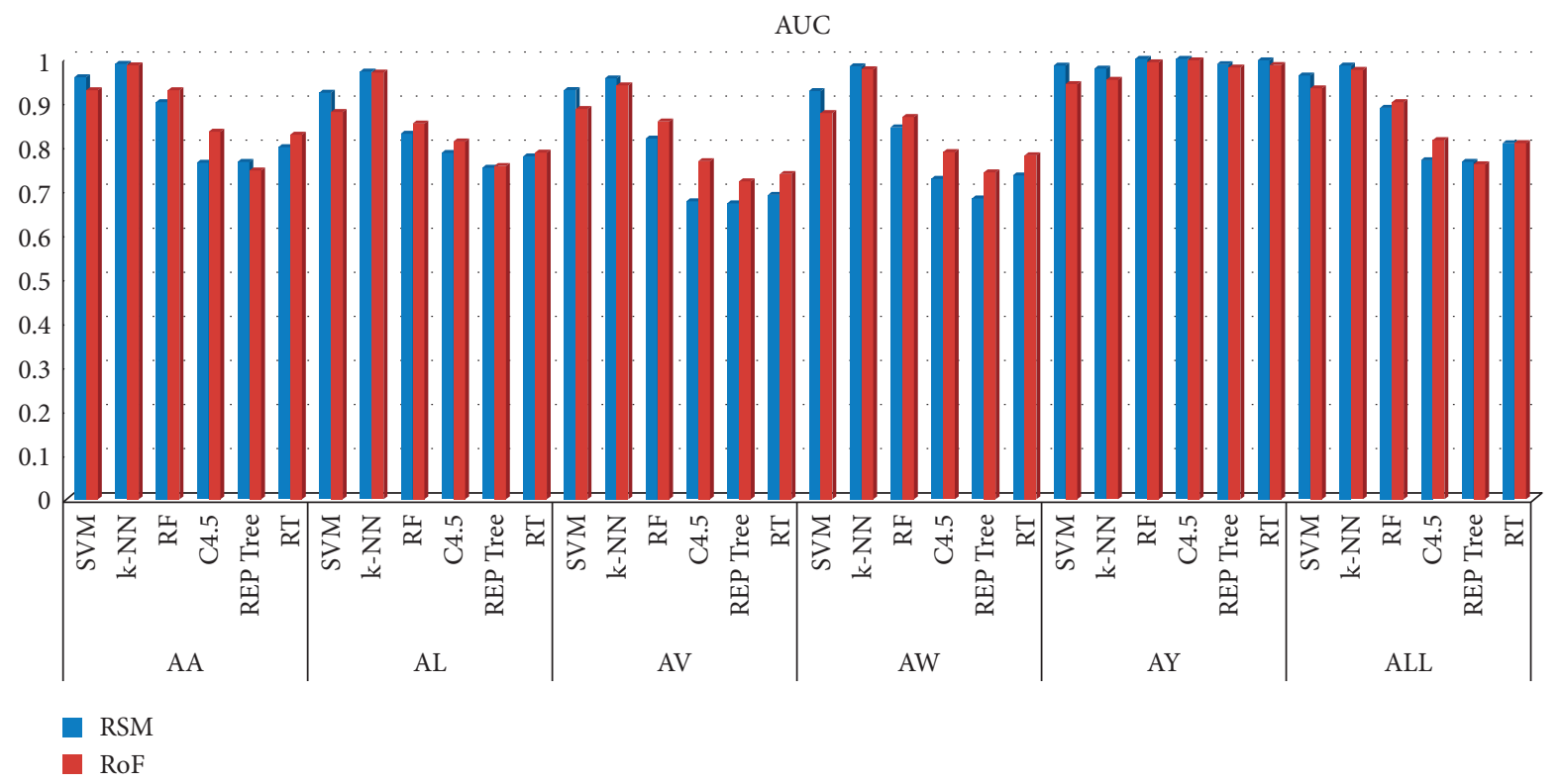

Figure 8: AUC of different classifiers.

TABle 2: Comparison with previous studies.

\begin{tabular}{lcccc}
\hline Study & Feature extraction & Classifier & Classes/subject(s) & Accuracy (\%) \\
\hline$[18]$ & Rényi min-entropy & RF & $4 /$ subject independent & 80.55 \\
{$[21]$} & Subbands PSDs & DNN & $2 /$ subject independent & 82.48 \\
{$[37]$} & Tangent space mapping & SVM & $2 / 1$ subject & 97.80 \\
{$[38]$} & Common spatial pattern & Backpropagation & $2 /$ subject independent & 80.73 \\
{$[39]$} & Neural network & SVM & $2 /$ subject independent & 91.9 \\
{$[40]$} & Regularized common spatial pattern & SVM & $2 /$ subject independent & 89.13 \\
{$[41]$} & Fisher ratio of time domain parameters & SVM & $2 /$ subject independent & 85.01 \\
{$[42]$} & Common spatial pattern & CNN & $2 /$ subject independent & 82.00 \\
{$[43]$} & Stacked autoencoders (SAE) & CNN & $2 /$ subject independent & 90.50 \\
{$[44]$} & Inverse problem through beamforming & Linear SVM & $2 /$ subject independent & 88.46 \\
Proposed & RF and RSM & $2 /$ subject dependent & 98.69 \\
& WPD & k-NN and RoF & $2 /$ subject independent & 94.83 \\
\hline
\end{tabular}


this aspect can be investigated. The developed system has the potential to be integrated into the future generation of BrainComputer Interface systems. The solution performed well for the intended dataset. Future work is to test its applicability for other potential Motor Imagery datasets. The incorporation of deep learning tools is another axis to explore.

\section{Conclusion}

In this paper, a novel automated Motor Imagery tasks classification method is proposed. The EEG signals are processed to distinguish between two categories of the brain activities. This approach is an intelligent combination of ensemble learning, Wavelet Packet Decomposition, Multiscale Principal Component Analysis, and subbands statistical features extraction. Results have shown its effectiveness in classifying the intended Motor Imagery tasks. Using an intelligent ensemble of the Random Subspace classifier with Random Forest, the highest subject-dependent accuracy of $98.69 \%$ is realized. The suggested ensemble of the Rotation Forest classifier with k-NN achieved the highest subjectindependent accuracy of $94.83 \%$.

\section{Data Availability}

The dataset used in this paper is publicly available via the following link: http://www.bbci.de/competition/iii/ desc_IVa.html.

\section{Conflicts of Interest}

The authors declare that there are no conflicts of interest regarding the publication of this study.

\section{Acknowledgments}

This project was funded by Effat University with Decision no. UC\#7/28 Feb. 2018/10.2-44i.

\section{References}

[1] S. Sanei, Adaptive Processing of Brain Signals, John Wiley \& Sons, Hoboken, NJ, USA, 2013.

[2] W. Gao, J.-a. Guan, J. Gao, and D. Zhou, "Multi-ganglion ANN based feature learning with application to P300-BCI signal classification," Biomedical Signal Processing and Control, vol. 18, pp. 127-137, 2015.

[3] C. Guger, S. Daban, E. Sellers et al., "How many people are able to control a P300-based brain-computer interface (BCI)?” Neuroscience Letters, vol. 462, no. 1, pp. 94-98, 2009.

[4] B. Z. Allison, E. W. Wolpaw, and J. R. Wolpaw, "Braincomputer interface systems: progress and prospects," Expert Review of Medical Devices, vol. 4, no. 4, pp. 463-474, 2007.

[5] J. R. Wolpaw, N. Birbaumer, D. J. Mcfarland, G. Pfurtscheller, and T. M. Vaughan, "Brain - computer interfaces for communication and control," vol. 113, pp. 767-791, 2002.

[6] C. U. Neuper and G. Pfurtscheller, "Event-related dynamics of cortical rhythms: frequency-specific features and functional correlates," International Journal of Psychophysiology, vol. 43, no. $1,2001$.
[7] A. Vasilyev, S. Liburkina, L. Yakovlev, O. Perepelkina, and A. Kaplan, "Assessing motor imagery in brain-computer interface training: psychological and neurophysiological correlates," Neuropsychologia, vol. 97, pp. 56-65, 2017.

[8] N. Mora, I. De Munari, and P. Ciampolini, A Multi-Modal BCI System for Active and Assisted Living, Springer, New York, NY, USA, 2016.

[9] R. Zarei, J. He, S. Siuly, and Y. Zhang, "A PCA aided crosscovariance scheme for discriminative feature extraction from EEG signals," Computer Methods and Programs in Biomedicine, vol. 146, pp. 47-57, 2017.

[10] T. Kayikcioglu and O. Aydemir, "A polynomial fitting and k-NN based approach for improving classification of motor imagery BCI data," Pattern Recognition Letters, vol. 31, no. 11, pp. 1207-1215, 2010.

[11] B. Blankertz, K.-R. Müller, D. J. Krusienski et al., “The BCI competition III: validating alternative approaches to actual BCI problems," IEEE Transactions on Neural Systems and Rehabilitation Engineering, vol. 14, no. 2, pp. 153-159, 2006.

[12] D. J. Leamy, J. Kocijin, K. Domijan et al., "An exploration of EEG features during recovery following stroke-implications for BCI-mediated neurorD. R. Collins, ehabilitation therapy," J. Neuroengineering Rehabil, vol. 11, no. 1, p. 9, 2014.

[13] D. Li, H. Zhang, M. S. Khan, and F. Mi, "A self-adaptive frequency selection common spatial pattern and least squares twin support vector machine for motor imagery electroencephalography recognition," Biomedical Signal Processing and Control, vol. 41, pp. 222-232, 2018.

[14] J. Kevric and A. Subasi, "Comparison of signal decomposition methods in classification of EEG signals for motor-imagery BCI system," Biomedical Signal Processing and Control, vol. 31, pp. 398-406, 2017.

[15] M. Miao, A. Wang, and F. Liu, "Application of artificial bee colony algorithm in feature optimization for motor imagery EEG classification," Neural Computing \& Applications, vol. 30, pp. 1-15, 2017.

[16] H. Baali, A. Khorshidtalab, M. Mesbah, and M. J. Salami, “A transform-based feature extraction approach for motor imagery tasks classification," IEEE J. Transl. Eng. Health Med, vol. 3, pp. 1-8, 2015.

[17] S. Chaudhary, S. Taran, V. Bajaj, and S. Siuly, "A flexible analytic wavelet transform based approach for motor-imagery tasks classification in BCI applications," Computer Methods and Programs in Biomedicine, vol. 187, Article ID 105325, 2020.

[18] M. A. Rahman, F. Khanam, M. Ahmad, and M. S. Uddin, "Multiclass EEG signal classification utilizing Rényi minentropy-based feature selection from wavelet packet transformation," Brain Inform, vol. 7, no. 1, pp. 1-11, 2020.

[19] S. K. Khare and V. Bajaj, "A facile and flexible motor imagery classification using electroencephalogram signals," Computer Methods and Programs in Biomedicine, vol. 197, Article ID 105722, 2020.

[20] Y. You, W. Chen, and T. Zhang, "Motor imagery EEG classification based on flexible analytic wavelet transform," Biomedical Signal Processing and Control, vol. 62, Article ID 102069, 2020.

[21] S. Tiwari, S. Goel, and A. Bhardwaj, "MIDNN-a classification approach for the EEG based motor imagery tasks using deep neural network," Applied Intelligence, pp. 1-20, 2021.

[22] Y. K. Musallam, N. I. Alfassa, G. Muhammad et al., "Electroencephalography-based motor imagery classification using temporal convolutional network fusion," Biomedical Signal Processing and Control, vol. 69, Article ID 102826, 2021. 
[23] I. Daubechies, Different Perspectives on Wavelets, American Mathematical Soc., Providence, Rhode Island, 2016.

[24] W. Y. Yang, W. Cao, T.-S. Chung, and J. Morris, Applied Numerical Methods Using MATLAB, John Wiley \& Sons, Hoboken, NJ, USA, 2020.

[25] M. T. Sadiq, X. Yu, Z. Yuan, F. Zeming, A. U. Rehman, and I. Ulla, "Motor imagery EEG signals decoding by multivariate empirical wavelet transform-based framework for robust brain-computer interfaces," IEEE Access, vol. 7, pp. 171431-171451, 2019.

[26] A. Subasi and S. M. Qaisar, "Surface EMG signal classification using TQWT, Bagging and Boosting for hand movement recognition," Journal Ambient Intelligence Humanized Computing, 2020.

[27] E. Alickovic, J. Kevric, and A. Subasi, "Performance evaluation of empirical mode decomposition, discrete wavelet transform, and wavelet packed decomposition for automated epileptic seizure detection and prediction," Biomedical Signal Processing and Control, vol. 39, pp. 94-102, 2018.

[28] R. R. Bouckaert, E. Frank, M. Hall et al., WEKA Manual for Version 3-9-1, Univ. Waikato Hamilt. N. Z., Hamilton, New Zealand, 2016.

[29] A. Subasi, Practical Guide for Biomedical Signals Analysis Using Machine Learning Techniques: A MATLAB Based Approach, Academic Press, Cambridge, MA, USA, 2019.

[30] X. Wu, V. Kumar, J. R. Quinlan et al., “Top 10 algorithms in data mining," Knowledge and Information Systems, vol. 14, no. 1, pp. 1-37, 2008.

[31] M. Hall, I. Witten, and E. Frank, Data Mining: Practical Machine Learning Tools and Techniques, Kaufmann Burlingt, Burlington, MA, USA, 2011.

[32] J. Han, J. Pei, and M. Kamber, Data Mining: Concepts and Techniques, Elsevier, Amsterdam, Netherlands, 2011.

[33] J. J. Rodriguez, L. I. Kuncheva, and C. J. Alonso, "Rotation forest: a new classifier ensemble method," IEEE Transactions on Pattern Analysis and Machine Intelligence, vol. 28, no. 10, pp. 1619-1630, 2006.

[34] T. K. Ho, "The random subspace method for constructing decision forests," IEEE Transactions on Pattern Analysis and Machine Intelligence, vol. 20, no. 8, pp. 832-844, 1998.

[35] M. Skurichina and R. P. Duin, "Bagging, boosting and the random subspace method for linear classifiers," Pattern Analysis \& Applications, vol. 5, no. 2, pp. 121-135, 2002.

[36] J. Y. Choi, D. H. Kim, K. N. Plataniotis, and Y. M. Ro, "Classifier ensemble generation and selection with multiple feature representations for classification applications in computer-aided detection and diagnosis on mammography," Expert Systems with Applications, vol. 46, pp. 106-121, 2016.

[37] M. Sartori, S. Fiori, and T. Tanaka, "An experimental study to compare CSP and TSM techniques to extract features during motor imagery tasks," Signal Process. Mach. Learn. Brain-Mach. Interfaces, 2018.

[38] H. Jia, S. Wang, D. Zheng, X. Qu, and S. Fan, "Comparative study of motor imagery classification based on BP-NN and SVM," Journal of Engineering, no. 23, pp. 8646-8649, 2019.

[39] J. Jin, Y. Miao, I. Daly, C. Zuo, D. Hu, and A. Cichocki, "Correlation-based channel selection and regularized feature optimization for MI-based BCI," Neural Networks, vol. 118, pp. 262-270, 2019.

[40] Y. Park and W. Chung, "Selective feature generation method based on time domain parameters and correlation coefficients for Filter-Bank-CSP BCI systems," Sensors, vol. 19, no. 17, p. $3769,2019$.
[41] S. Selim, M. M. Tantawi, H. A. Shedeed, and A. Badr, "A CSP $\backslash$ AM-BA-SVM approach for motor imagery BCI system," IEEE Access, vol. 6, pp. 49192-49208, 2018.

[42] R. Arabshahi and M. Rouhani, "A Convolutional neural network and stacked autoencoders approach for motor imagery based brain-computer Iinterface," in Proceedings of the 2020 International Conference on Computer of Knowledge Engineering (ICCKE), pp. 295-300, Mashhad, Iran, October 2020.

[43] C. Ieracitano, N. Mammone, A. Hussain, and F. C. Morabito, "A novel explainable machine learning approach for EEGbased brain-computer interface systems," Neural Computing \& Applications, pp. 1-14, 2021.

[44] H. Varsehi and S. M. P. Firoozabadi, "An EEG channel selection method for motor imagery based brain-computer interface and neurofeedback using Granger causality," Neural Networks, vol. 133, pp. 193-206, 2021.

[45] S. M. Qaisar and A. Subasi, "Cloud-based ECG monitoring using event-driven ECG acquisition and machine learning techniques," Phys. Eng. Sci. Med, vol. 43, no. 2, pp. 623-634, 2020.

[46] S. M. Qaisar and A. Subasi, "Effective epileptic seizure detection based on the event-driven processing and machine learning for mobile healthcare," J. Ambient Intell. Humaniz. Comput, no. 1-13, 2020.

[47] S. Mian Qaisar, "Event-driven coulomb counting for effective online approximation of Li-ion battery state of charge," Energies, vol. 13, no. 21, p. 5600, 2020.

[48] S. Mian Qaisar, "Signal-piloted processing and machine learning based efficient power quality disturbances recognition," PloS One, vol. 16, no. 5, Article ID e0252104, 2021. 